\title{
Design of Porous MOFs for gas storage applications
}

\author{
Sandeep Singh Dhankhar ${ }^{1}$, C. M. Nagaraja ${ }^{1}$ \\ ${ }^{1}$ Department Of Chemistry, I.I.T. Ropar, Rupnagar, India \\ E-mail: sandeepsingh@iitrpr.ac.in
}

Metal-organic frameworks (MOFs) are a new class of crystalline solids which are attracting growing interest not only due to their fascinating capability to form diverse structural architectures but also for their potential applications.[1] Owing to their high surface areas and tailored pore size and functionality, MOFs are gaining considerable attention as prospective candidates for hydrogen storage and selective carbon dioxide capture applications.[2] Since, $\mathrm{H} 2 \mathrm{has}$ been considered as an eco-friendly alternative to fossil fuels and several MOFs exhibiting very high $\mathrm{H} 2$ uptake properties at low temperatures ( $77 \mathrm{~K}$ ) have been reported. However, the maximum uptake achieved at ambient conditions is still close to $2-3 \%$ due to weak interaction of $\mathrm{H} 2$ (Qst $<10 \mathrm{~kJ} / \mathrm{mol})$ with the framework. To enhance the enthalpy of $\mathrm{H} 2$ adsorption (Qst) with MOFs several strategies have been investigated. In this context, we developed few MOFs with pores functionalized with polar groups such as, $\mathrm{F}-,-\mathrm{NH} 2$ to induce selective adsorption properties to the framework.[3] Further, MOFs composed of unsaturated metal ions exhibiting high enthalpy of $\mathrm{H} 2$ adsorption (Qst $=11.8 \mathrm{~kJ} / \mathrm{mol}$ ) have also been developed using green synthetic techniques such as, mechanochemical and sonochemical routes, these results will be presented.

[1] Cui Y. et al. (2016). Acc. Chem. Res., 49, 483-493.

[2] Ugale B. et al. (2016) Inorg. Chem., 55, 9757- 9766.

[3] Dhankhar S. S. et al. (2015). Eur. J. Inorg. Chem., 34, 5669-5676.

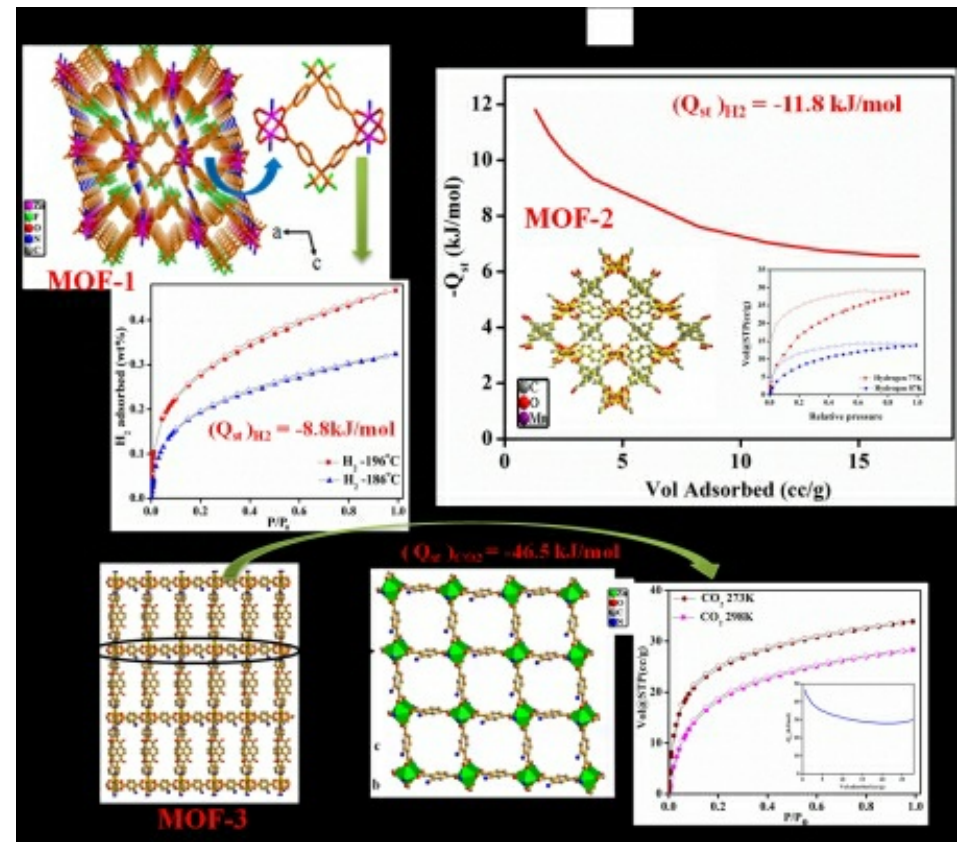

Keywords: Porous MOFs, Green synthesis, Selective gas adsorption 